\title{
POINTING CORRECTION IN AN ADAPTIVE ARRAY FOR COMMUNICATIONS
}

\author{
Miguel A. Lagunas, J. Fernandez, M. Najar
}

ETSI de Telecomunlcaclon, Departamento de TSC

Apdo. 30002, 08080 BARCELONA, SPAIN

\section{ABSTRACT ${ }^{1}$}

This work deals with dsp procedures for pointing correction in adaptive arrays which use a spatial reference to form the optimum beam (GSLC). The problem of adequate pointing arises from many effects on the aperture and the receiver front-end of every array channel. Component aging, channel mismatching, sensor location, etc, promote steering errors that may produce the rejection of the desired signal. Currently, high complicated telemetry systems and monopulse radars are used for steering correction. This work describes the existing alternatives as well as further improvements that can be achieved from the use of temporal references combined with the GSLC beamformer.

\section{INTRODUCTION}

The increasing number of mobile communication systems and ground radar stations promotes the interest of adaptive array beamforming for on-board satellite communications.

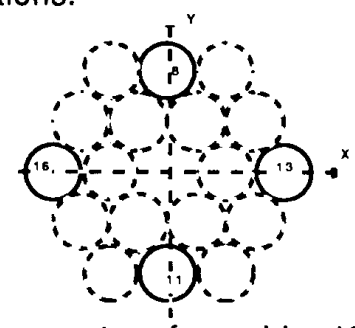

Fig 1. The aperture formed by 18 sensors

1 This work was supported by the National Research Plan of Spain under project DGTTIC302, and in part by the CIRIT of Catalunya with grant BV91/1-56
Because the desired direction is known, the adaptive array takes the form of a Generalised Sidelobe Canceller (GSLC). In order to specify the problem, all the experiments supporting this work were done over the so-called MAM antenna. The aperture is formed by 18 sensors located as depicted in fig1, working at a central frequency of $19 \mathrm{Ghz}$ and with a field of view of $+/-10^{\circ}$.

The GSLC was formed by a phased array as quiescent with the corresponding blocking matrix in the lower branch. The adaptive algorithm for the adaptive weights in the lower branch is Kalman type with square-root propagation of the covariance matrix.

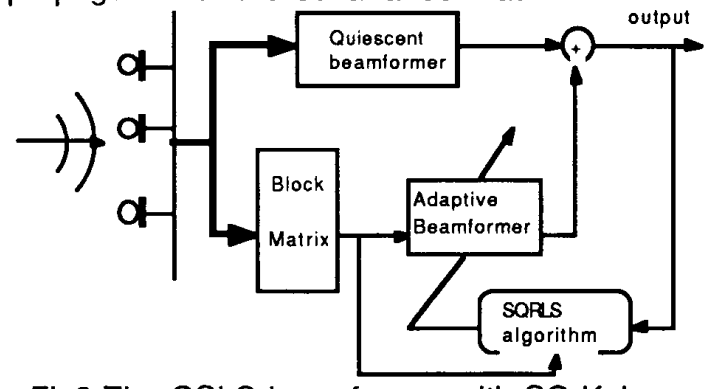

Fig2 The GSLC beamformer with SQ-Kalman adaptive algorithm

The square root version was able to support hard quantization in the overall beamformer, being 6 bits for the snapshots and progressive bit assignment in three layers of Asics from 4 up to 16 bits in the GSLC output. The residual in the diagonal of the square-root matrix recursion was set to $10 \mathrm{e}$ $4 / 1 /$ in order to allow tracking of interferers with radial velocity of $0.1^{\circ}$ per second.

The first problem, related with pointing, comes from the requirement of automatic steering correction from earth stations. These corrections were usually carried out by 
corrections were usually carried out by telemetry to a monopulse radar in the satellite. Of course, changing the desired direction in the above structure will motivate the change of both quiescent and blocking matrix. This operation was out of the possibilities of the inboard processor. In order to avoid the mentioned problem, a pre-steering matrix has to be added to the old GSLC structure.

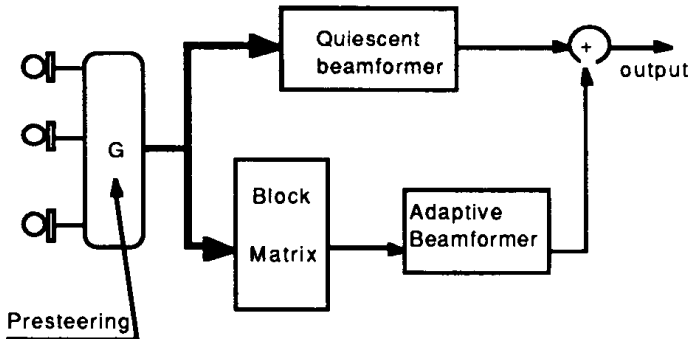

Fig 3 The GSLC structure with pre-steering matrix

The pre-steering stage can be characterised by a diagonal matrix $\mathbf{G}$ which contains just the phase shifts to convert any direction $\left(\Theta_{0}, \Phi_{0}\right)$ to the aperture broadside. Regardless this matrix will play a very important role in the rest of this work, it is important to remark that it is almost mandatory for GSLCs in satellite payloads. The reason is that, even in the case of non-mobile scenarios, missmatching between receivers front-ends, down-conversion and sensor missfunctioning, will produce the same problem demanding for re-steering the array. Just to give an idea, the following table provides the loss in signal to interference ratio (in $\mathrm{dBs}$ ), for the above aperture, when the desired signal is a BPSK of 3 Mbps ($12.55 \mathrm{~dB}$ which corresponds to Spread Spectrum modulation systems) and an unmodulated interference is located at $3^{\circ}$ from it with $17.45 \mathrm{dBs}$. for phase missmatching and 27.44 for phase missmatching.

magnitude miss. CIR phase miss. CIR

$\begin{array}{llll}0.0 & 17.32 & 0 \% & 29.8 \\ 1.0 & 14.91 & 1 \cong & 21.1 \\ 2.0 & 16.75 & 2 \varrho & 15.6\end{array}$

Note how phase missmatching almost twice loss (in $d B$ ) that magnitude missmatching. The effect is that whenever the desired signal impinges the aperture more than $2 \circ$ from the steered direction the GSLC starts to look at it as an interference. In fact for phase missmatching close to $2.3^{\circ}$ the desired signal is almost nulled out by the algorithm. The values given for mismatch reflect the standard deviation in $\mathrm{dBs}$ of a gaussian distribution for the sensors gain around their nominal value; and, in the case of phase, the listed values correspond to the maximum of an uniform phase distribution around the corresponding nominal values.

The aim of this work was to use the presteering matrix elements in order to track the desired signal either for misspointing of the array or beacuse it is moving with radial velocity.

\section{TIME REFERENCE CONTROL OF A GSLC} BEAMFORMER

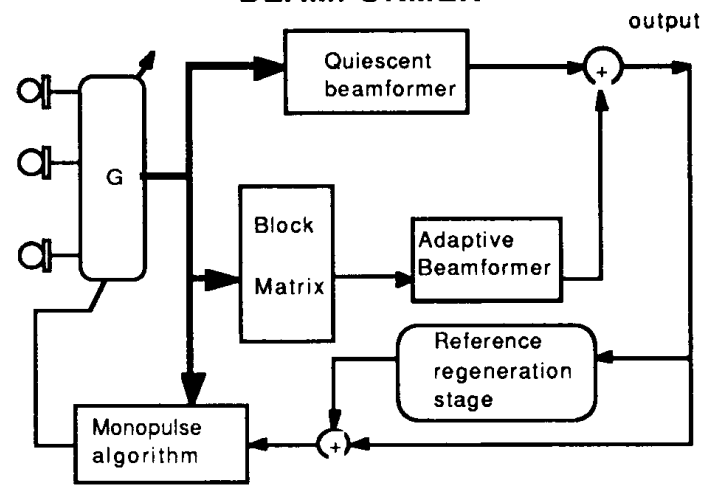

Fig. 4 GSLC with time reference pre-steering

From the above section, a new structure culd be proposed. This structure, in addition to the GSLC loop which works independently of the steering angle, includes a second loop, with lower rate than the GSLC loop, which exploits the time evolution of the desired signal in order to keep steered the overall beamformer response. The structure is shown in figure 4 .

The problem we are going to face on this work is the potential misspointing of the presteering block with respect the actual doa of the desired source.

The first alternative consist in the use of DOA estimation techniques in order to locate the desired source, assuming it is nearby the 
alternative is that DOA estimates do not identify the desired source with respect interferences, since they are not sensitive to modulation parameters on the impinging energy; also, the scanning in elevation and azimuth of the DOA estimate could be very time consuming for the satellite payload.

A second approach, within the structure depicted in figure 4 , is to set a parallel beamformer with temporal reference which provides the corresponding pointing angles to the GSLC pre-steering.

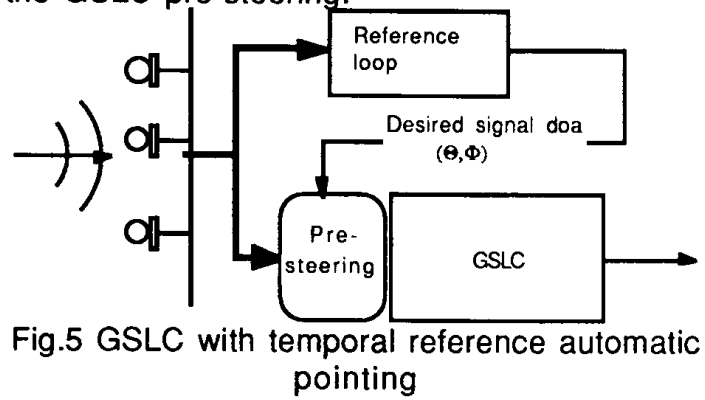

Regardless of important issues like reference framing, code access, modulation and protocols, seems to be that if the reference loop is able to regenerate the desired signal the reference loop may provide the adequate steering direction to the GSLC. Of course, in order to keep the communication link the reference loop has to be framed in time (TDM) or in frequency (FDM) with the information channel managed by the GSLC.

The most suggestive approach is to include a coded BPSK signal as temporal reference in the upper zone of the communication bandwidth, as it is indicated in figure 6 .

Note that whenever in the upper loop there is not interferences, the temporal reference algorithm will steer the desired signal, and the corresponding weights can be copied directly to the diagonal elements of the pre-steering matrix. The assumption that no significant interferences are present in the temporal reference loop is crucial and it depends very much on the narrowbandwidth assigned to this loop.

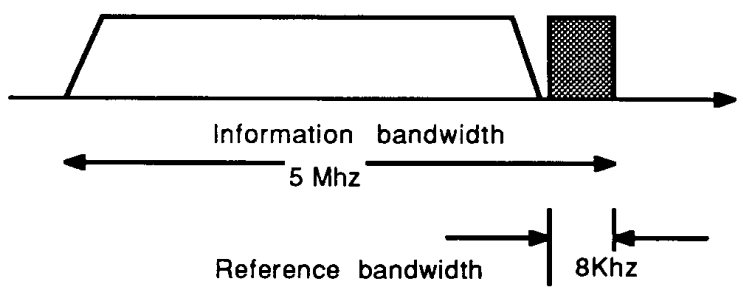

Fig. 6.- $8 \mathrm{KHz}$ reference for steering purposes (reference loop) inside the information bandwidth of the communication link (GSLC bandwidth)

The main drawback of this scheme is that the presence of interferences in the steering loop promotes the same problems of pointing we have in the GSLC rendering the problem back to the initial situation. Just to give an idea in figure 7 is depicted the response of a temporal reference loop /2/ where the reference is found at $0^{\circ}$ in presence of a severe jammer located in the mainlobe.

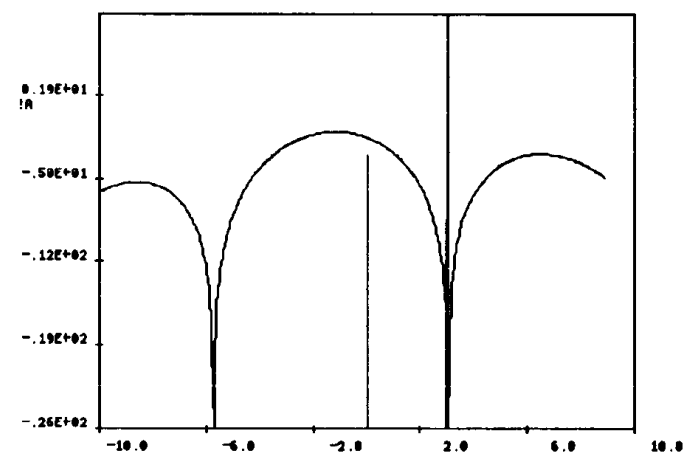

Fig. 7 Temporal reference loop response to a desired signal, found at $0^{\circ}$ in presence of a severe jammer at $3^{\text {o }}$

As the reader can see, the presence of interferers in the reference loop introduces bias in the beamformer rendering non valid the overall structure. Furthermore, even in the case of secondary lobe interferences, the pointing is correct but the weights cannot be copied to the diagonal of the pre-steering matrix because it is not sure that the fixed blocking matrix remains valid for the presteered snapshot. In summary the temporal reference loop will be adequate whenever the reference is framed in time or frequency in such a way the only it will be present in the temporal reference loop. This situation that may be common in some mobile 
communications scenarios, fails in the case of collision when two users demand for a dedicated beam simultaneously.

Finally, the authors $/ 3 /$ and L.J.Griffiths /4/ develop a system which exploits the constant envelope associated to the most used modulations for the desired signal. The basic idea is to control the pre-steering angle in such a way that the envelope or shorttime power at the array output is maximised. The algorithm could be a DSD /5/ random search or monopulse. In any case, random search is highly recommended. In the random search a random perturbation is promoted in elevation and azimuth, if the envelope or the short-time power of the output increases the perturbation is retained in the steering angles; if not the steering remains the same. A version of monopulse, handling the output of a short time RMS detector, with symmetric spatial perturbations, was used by the authors with success in data relay satellite scenarios (DRS), where the desired signal impinges the array with SNRs above $3 \mathrm{~dB}$ at the sensor level.

In any case, the main problem of these procedures resides in the cases that very low SNRs, at the sensor level, has to be managed. As an example, a BPSK to be detected with a bit error rate of $1 \mathrm{e}-4$ requires an EbNo of $8.7 \mathrm{~dB}$ at the GSLC output; this means that at the sensor level will be around $-3.2 \mathrm{~dB}$. With these low signal levels, the envelope is almost independent, due the the output noise, to steering movements of $1^{\circ}$ which are enough to promote the rejection of the desired signal by the mispointed GSLC. The mentioned situation becomes more severe when spread spectrum is the original modulation for the received BPSK signal, being the input SNR in the range of $10 \mathrm{db}-5 \mathrm{dBs}$. None of the previously described techniques may cope this case.

We can say that the reason why the described techniques cannot cover the case of many practical systems in communications applications is due to the fact that working only over the envelope does not provide any modulation or bandwidth compression gain in the reference analysis. Next section will report how the use of good estimates of the desired signal waveform can improve a lot the dynamic range covered by the envelope techniques.

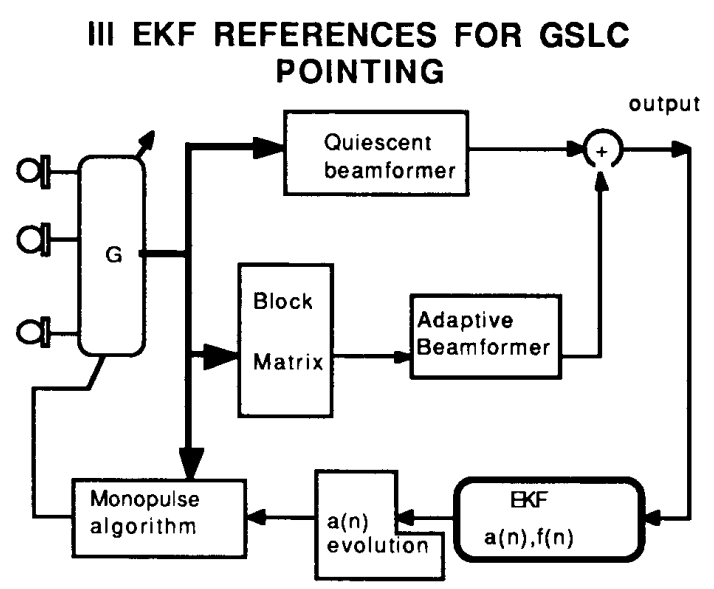

Fig. 8. The EKF loop for pre-steering

The scheme proposed is a slight derivation of the structure shown in figure 4 . In the new scheme an Extended Kalman Filter (EKF) has been used to acquire and track the desired signal from the GSLC output. The EKF estimates the magnitude, instantaneous phase and instantaneous frequency of the GSLC output. Tracking the magnitude, almost free of noise, the presteering angle is corrected with the monopulse principle over a random perturbation in elevation and azimuth. At the same time, tracking the instantaneous phase and frequency, the pointing system is able to cope with doppler effects in the received frequency and with the radial velocity of the desired user.

The main advantage of the EKF in following the carrier evolution is that it is able to track $0 \mathrm{~dB}$ carriers at the output (almost -10 dB level at the sensor level) with $1 \%$ error over the actual magnitude value. Doing this, together with the frequency tracking the systems reaches levels of performance unexpected from the previous schemes. Just to provide some insight in the EKF performance, the following table shows the frequency tracking error for different SNR at the array output. The residual mentioned in the table refers to the diagonal value, the same for magnitude phase and frequency, of the 
covariance matrix propagation equation involved in the EKF /6/.

\begin{tabular}{|c|c|c|c|c|}
\hline SNR dB & & \multicolumn{3}{|c|}{ Residual Q } \\
\hline \multirow{3}{*}{-5.5} & & 0.001 & 0.0001 & 0.00001 \\
\hline & Convergence & no & no & no \\
\hline & Tracking error & no & no & no \\
\hline \multirow{2}{*}{-1.5} & Convergence & 10 & 10 & no \\
\hline & Tracking error & -6.56 & -12.48 & no \\
\hline \multirow{2}{*}{2.5} & Convergence & 10 & 10 & no \\
\hline & $\begin{array}{l}\text { Tracking orror } \\
\text { in samples }\end{array}$ & -6.64 & -12.40 & no \\
\hline \multirow{2}{*}{6.5} & Convergence & 20 & 25 & no \\
\hline & $\begin{array}{l}\text { Tracking error } \\
\text { in samples }\end{array}$ & .6 .64 & -12.40 & no \\
\hline \multirow{2}{*}{10.5} & Convergence & 20 & 25 & no \\
\hline & $\begin{array}{l}\text { Tracking error } \\
\text { in samples }\end{array}$ & -6.64 & -12.32 & no \\
\hline \multirow{2}{*}{13.5} & Convergence & 20 & 25 & $>1000$ \\
\hline & $\begin{array}{l}\text { Tracking error } \\
\text { in samples }\end{array}$ & -6.60 & -12.26 & $>3$ \\
\hline
\end{tabular}

Note, from this table that the system has convergence rates below 50 snapshots, being the doppler of $1 \mathrm{Khz}$ and the doppler rate of $1 \mathrm{Khz}$ per second. This doppler rate is far above of the usual $50 \mathrm{~Hz}$ per second associated with the scenarios used in the application under the scope of this work. The following figures show the EKF performance in frequency tracking.

From this figure is clear that the monopulse explorations in elevation and azimuth can be done every 50 snapshots or more per perturbation without paying attention to the problems derived from time varying position, doppler and other problems. At the same time, the tracking of magnitude fluctuations done at $8 \mathrm{dBs}$ (required for BPSK) at the output is done almost independent of output noise and interference residuals. Experiences carried out by the authors ensures acquisition of adequate steering even for mispointing close to $1 \%$. Regardless that with these errors the aperture may reject in 5 $\mathrm{dB}$ or $10 \mathrm{~dB}$ the desired signal, the excellent performance of the EKf in regenerating the carrier allows the pointing correction after 1000 snapshots at the sampling level of the EKF bandwidth, which is $8 \mathrm{KHs}$, meaning about
$125 \mathrm{msec}$. to correct such steering error. With respect to tracking desired sources with radial velocity, satisfactory results have been obtained for sources with radial velocity of $2^{\circ}$ per second. The tracking error is lower than $0.05^{\circ}$ for this case. Nevertheless, the authors found that the most critical part in this design, before the implementation of the breadbording was the adjustment of the monopulse parameters like size of perturbation, size of the correction and average time to validate the magnitude values to perform the update of the steering.

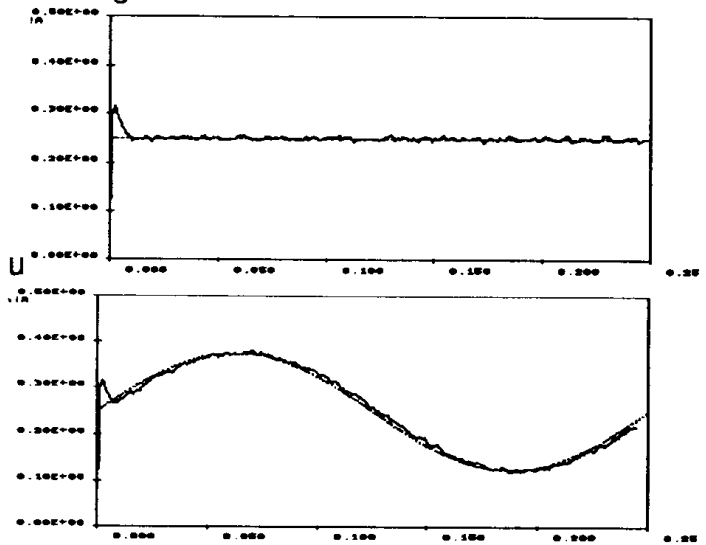

Fig 9. EKF acquisition for $0 \mathrm{db}$

(top)Acquisition and tracking for a doppler rate of $1 \mathrm{Khz}$ per second.

\section{REFERENCES}

|1|J.Fernandez,M.Lagunas, G.Vazquez,"Breadbordin $\mathrm{g}$ of a digital beamforming network" ERA-ESA Technical report, European Spatial Agency, Doc. \#1897/01. Contract 8714/90/NL/US(SC), The Netherlands, Dec 1990

12) M.A. Lagunas. "Direction of Arrival Techniques and Temporal Beamforming" European Spatial Agency, ESA-report,P.O. 112052, October 1991, The Netherlands.

(3) J.Fernandez, "Adaptive beamforming with temporal and spatial references in satellite communications", Signal Proc. V, Eusipco 90, Ed. M.A.Lagunas, ELSEVIER, pp.733-736, Sept 1990.

14| L.J. Griffiths. "New results in constrained beamforming. Non linear constraint and constant modulus output". Signal Proc. V, Eusipco 90, Ed. M.A.Lagunas, ELSEVIER, pp. 1-8, Sept 1990.

14) R.A. Monzingo,T.W. Miller. "Introduction to adaptive arrays". N.Y., Wiley, 1980. 